\title{
Eco-Friendly Fabrication of Plasmonically Active Substrates Based on End-Grafted Poly(ethylene glycol) Layers
}

\author{
Sema Karabel Ocal, ${ }^{\dagger \neq}$ Sami Pekdemir, ${ }^{\dagger \neq}$ Murat Serhatlioglu, ${ }^{\S}$ Hasan H. Ipekci, ${ }^{\dagger, \dagger}$
}

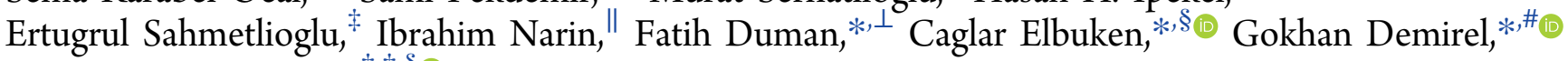
and M. Serdar Onses $*,+,, \S_{(0)}$

\author{
${ }^{\dagger}$ Department of Materials Science and Engineering, Erciyes University, 38039 Kayseri, Turkey \\ ${ }^{\ddagger}$ Nanotechnology Research Center (ERNAM), Erciyes University, 38039 Kayseri, Turkey \\ ${ }^{\S}$ UNAM-National Nanotechnology Research Center, Institute of Materials Science and Nanotechnology, Bilkent University, 06800 \\ Ankara, Turkey \\ "Faculty of Pharmacy, Erciyes University, 38039 Kayseri, Turkey \\ ${ }^{\perp}$ Department of Biology, Faculty of Science, Erciyes University, 38039 Kayseri, Turkey \\ \#Bio-Inspired Materials Research Laboratory (BIMREL), Department of Chemistry, Gazi University, 06500 Ankara, Turkey
}

\section{Supporting Information}

ABSTRACT: We report completely sustainable processes and materials for inexpensive and scalable fabrication of plasmonically active solid substrates, which are critical for emerging applications in sensing, catalysis, and metasurfaces. Our approach involves grafting of poly(ethylene glycol) (PEG) onto silicon oxide terminated solid substrates using all-water based processing leading to an ultrathin $(12 \mathrm{~nm})$ and smooth (roughness of $\sim 1 \mathrm{~nm}$ ) functional layer. The resulting

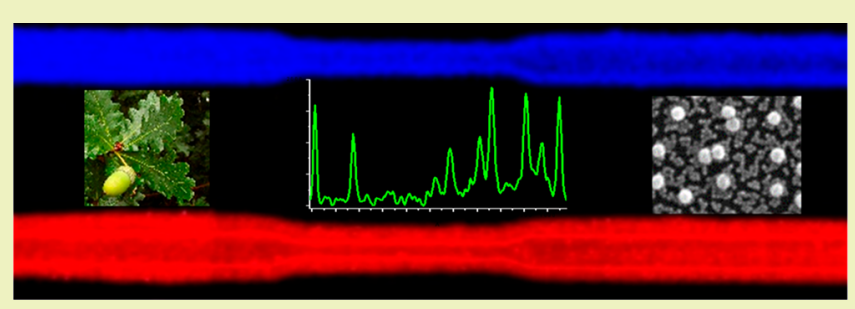
surfaces facilitate robust and effective immobilization of gold nanoparticles (NPs) with a density that is superior to the organic solvent based processing. This new process achieves size dependent assembly of the citrate-stabilized gold NPs resulting in high plasmonic activity in surface-enhanced Raman scattering (SERS). The use of leaf extracts derived from Quercus pubescens as a reducing and stabilizing agent allowed for green synthesis of gold NPs with an average diameter of $25.6 \pm 11.1 \mathrm{~nm}$. The assembly of the green synthesized gold NPs on all-water processed PEG grafted layers enabled a fully sustainable route for fabrication of plasmonically active solid substrates. The resulting substrates exhibited high SERS response over the entire $(\sim 1$ $\mathrm{cm}^{2}$ ) substrate surface with an analytical enhancement factor of $9.48 \times 10^{4}$ for the probe molecule rhodamine $6 \mathrm{G}$ under $532 \mathrm{~nm}$ laser excitation. A microfluidic device was also constructed on the fabricated platform for SERS mediated simultaneous detection of two nonsteroidal anti-inflammatory drugs, dexketoprofen and ibuprofen, which are widely used in human medicine and present as contaminants in wastewater. The biocompatibility of PEG together with all-water based processing overcome the need for waste management and ventilation of the working place enabling cost and energy efficient, environmentally benign fabrication of plasmonic devices.

KEYWORDS: Green chemistry, Plasmonics, Functional surfaces, SERS, End-grafted poly(ethylene glycol)

\section{INTRODUCTION}

Plasmonic devices enable several important applications in sensing, ${ }^{1}$ catalysis, ${ }^{2}$ biotechnology, ${ }^{3}$ solar cells, ${ }^{4}$ and optoelectronics devices. ${ }^{5}$ Such applications rely on metallic nanostructures that are uniformly placed on a substrate. The basis of these devices is surface plasmons that can be tailored toward the specific application by modulating the properties (e.g., size, geometry, structure, composition) of the individual nanostructures and coupling between these structures as a function of their separation distance. ${ }^{6}$ The conventional route for fabrication of plasmonic devices involves preparation of templates via lithography combined with metal deposition/ etching. ${ }^{7,8}$ Despite the unmatched spatial control over the plasmonic nanostructures produced by nanofabrication, several issues including the cost, scalability, polycrystallinity, and roughness of the resulting metal nanostructures limit their utilization in practical applications. A critical concern about the large-scale fabrication of plasmonic devices via lithography relates the vast use of toxic chemicals including organic solvents, strongly acidic/basic solutions, and corrosive gases. The alternative route involves colloidal metal nanoparticles (NPs) that are massively available at desired properties through wet-chemistry methods. ${ }^{9}$ Colloidal metal NPs are often synthesized in aqueous solutions with limited usage of

Received: November 24, 2018

Revised: January 9, 2019

Published: January 14, 2019 
toxic chemicals. Moreover, the past decade witnessed the advent of a range of synthetic methods involving the use of natural chemicals in the green synthesis of NPs contributing to the sustainability of these processes. ${ }^{10-13}$ The fabrication of plasmonic devices based on colloidal NPs requires uniform and robust assembly of the particles on the substrates with the ability to tune the density of bound particles and interparticle distances. The immobilization of NPs is performed by chemical functionalization of the substrate, which requires the use of organic solvents and toxic chemicals. ${ }^{14-16}$ Therefore, it is highly desirable to develop environmentally benign, scalable, and low-cost platforms that enable uniform and robust assembly of colloidal NPs for fabrication of plasmonic devices.

Here we report environmentally benign materials and processes for versatile fabrication of functional surfaces to serve as templates in the immobilization of NPs into plasmonic assemblies. At the center of our platform is a biocompatible and water-soluble material, poly(ethylene glycol) (PEG), which is extensively used in industrial applications that range from medicine to cosmetics. ${ }^{17-19}$ Recently, we and others have demonstrated that when grafted onto substrates and patterned at the nanoscale, PEG interacts with gold NPs in unique ways to assemble the particles into configurations that are of significant interest for plasmonic applications. ${ }^{20-22}$ Despite the biocompatibility of the material itself, previous studies on the assembly of NPs on substrates functionalized with PEG involved the use of toxic organic solvents. ${ }^{23-25}$ Our objective in this study is to develop all-water based processes in fabrication of surfaces that are functionalized with PEG. The grafting of PEG onto the surface of the substrate is performed via one-step reaction through the hydroxyl end-groups of the molecule. This grafting scheme allows for the use of the industrially available form of the polymer without the need for tedious postfunctionalization steps leading to a low-cost and scalable process. Additionally, for the first time, we present assembly of green synthesized gold NPs that are prepared using natural reducing agents, on end-grafted PEG layers, leading to a completely sustainable process from the surface functionalization to NP synthesis. The systematic studies of synthesis allow for preparation of spherical gold NPs with a high size and shape uniformity. The spectroscopic characterization of the green synthesized gold NPs suggests that the presence of carboxylate groups is important for the stabilization of the particles and their interaction with the PEG-grafted substrates. The uniformity and functionality of the gold NP arrays immobilized on PEG grafted substrates are investigated with ex-situ SEM imaging and Raman mapping of a probe molecule (rhodamine 6G) in surface-enhanced Raman scattering (SERS). ${ }^{26}$ The assembled gold NPs result in a highly intense SERS response over the entire substrate and enable detection of analyte molecules down to 100 pM. Furthermore, we demonstrate microfluidic assisted SERS sensing of drug molecules as an application of the fabricated platform.

\section{EXPERIMENTAL SECTION}

Materials. Silicon wafers were purchased from Wafer World Inc. Spherical gold nanoparticles were purchased from TED Pella Inc. Leaves of Quercus pubescens (downy oak) were collected from Kayseri, Turkey. PEG $(35.0 \mathrm{~kg} / \mathrm{mol})$ was purchased from Polymer Source Inc. (polydispersity index $=1.15$ ). $\mathrm{HAuCl}_{4}$, rhodamine $6 \mathrm{G}$, methylene blue, chloroform (99.0\%), and chlorobenzene (99.0\%) were purchased from Sigma-Aldrich Inc. Sylgard 184 silicone elastomer kit from Dow Corning and SU-8 photoresist from MicroChem were used for fabrication of microfluidic channels. Dexketoprofen and ibuprofen were obtained from Deva Inc. and BASF Inc., respectively.

Preparation of PEG-Grafted Substrates and Assembly of NPs. Silicon wafers were first cut into $1 \times 1 \mathrm{~cm}^{2}$ pieces and then cleaned in a UV-ozone chamber for $20 \mathrm{~min}$. To graft PEG, a solution (6 wt \%, unless otherwise stated) of PEG in water was spin-coated on the freshly cleaned substrate. The spin-coating was performed at 3000 $\mathrm{rpm}$ for $30 \mathrm{~s}$ with an acceleration rate of $1000 \mathrm{rpm} / \mathrm{s}$. Afterward, the substrate was annealed at $180{ }^{\circ} \mathrm{C}$ under argon gas environment in a glovebox for $5 \mathrm{~min}$. To remove the ungrafted PEG chains, the substrate was washed in water under sonication for 3 cycles, $3 \mathrm{~min}$ each. Following the washing, the substrate was dried with nitrogen. Conventional grafting of PEG using organic solvents was also performed by spin-coating PEG from chlorobenzene (2 wt \%) at 3000 $\mathrm{rpm}$ for $30 \mathrm{~s}$ followed by washing in chloroform under sonication for 3 cycles, 3 min each. For the assembly of NPs, a solution of gold NPs was pipetted on the PEG grafted substrate and incubated for $1 \mathrm{~h}$. Finally, the substrate was washed in water for 2 min under sonication and then dried with nitrogen.

Green Synthesis of Gold NPs. The leaves of Quercus pubescens were cleaned with water followed by drying. In total, $10 \mathrm{~g}$ of the leaves was boiled in $100 \mathrm{~mL}$ of water and mixed with a magnetic stirrer for $20 \mathrm{~min}$. The obtained aqueous solution was then filtered (Whatman, no. 1) multiple times. The filtered solution of the extract was stored at $4{ }^{\circ} \mathrm{C}$ until use. A solution of $\mathrm{HAuCl}_{4}(20 \mathrm{~mL})$ at different concentrations $(0.1 \mathrm{mM}, 0.5 \mathrm{mM}, 1 \mathrm{mM})$ was heated until boiling, while stirring in a heating mantle. The aqueous solution of the extract $(0.25 \mathrm{~mL}, 0.50 \mathrm{~mL}, 0.75 \mathrm{~mL})$ was added quickly to the boiling solution. The color of the solution slowly turned into violet from petrol blue, indicating the reduction of gold ions. The aliquots of the solution were taken out at different times ( $30 \mathrm{~s}, 5 \mathrm{~min}, 15 \mathrm{~min})$ and then quenched at room temperature for UV-vis and TEM analysis.

Preparation of a Microfluidic Device and Delivery of Analyte Molecules. The microfluidic device was fabricated from polydimethylsiloxane (PDMS) using standard soft lithography procedures. $^{27,28}$ The mold was fabricated on a 4 -in. silicon wafer by SU-8 photoresist patterning. SU-8 2005 was used to obtain a channel height of $5 \mu \mathrm{m}$. Two different microchannel designs were prepared and transferred on a chrome/glass photomask. The first design consisted of four parallel channels with individual inlets and a common outlet. In the second design, all four channels had individual inlets and outlets. For the fabrication of the mold, first dehydration baking of silicon wafer was performed at $110{ }^{\circ} \mathrm{C}$ for $20 \mathrm{~min}$. Then, SU-8 was spin-coated at $2500 \mathrm{rpm}$ for $40 \mathrm{~s}$ to obtain the desired channel height. After soft baking at $65^{\circ} \mathrm{C}$ for $1 \mathrm{~min}$ and at $95^{\circ} \mathrm{C}$ for 3 min, the photoresist was UV exposed through the mask at a dose of $85 \mathrm{~mJ} / \mathrm{cm}^{2}$. Following the post baking process $\left(65^{\circ} \mathrm{C}\right.$ for $1 \mathrm{~min}, 95$ ${ }^{\circ} \mathrm{C}$ for $3 \mathrm{~min}$ ), the mold was developed for $1 \mathrm{~min}$ with gentle agitation. The fabrication of the mold was finalized by nitrogen drying of the wafer. For casting, 10:1 (w/w) mixed and debubbled PDMS solution was poured on the mold and cured at $80^{\circ} \mathrm{C}$ for $12 \mathrm{~h}$. Finally, PDMS microfluidic device was peeled off and inlet/outlet holes were generated by a biopsy punch. The microfluidic device was placed over the PEG grafted substrate with assembled gold NPs. The analyte molecules dissolved in ethanol were sucked from the channel inlet with the aid of vacuum applied from the outlet. The solvent evaporated slowly through the inlet region for $20 \mathrm{~min}$. The microfluidic device was then detached from the substrate.

Characterization. To measure the thickness of the end-grafted PEG layers, a stokes ellipsometer (Gaertner) with a fixed angle of incidence of $70^{\circ}$ was used. The thickness measurements were performed at three different locations of the substrate using a model consisting of silicon substrate/native silicon oxide layer (thickness of $1.5 \mathrm{~nm}$, refractive index of 1.543)/PEG (refractive index of 1.46). Absorption spectra of the green synthesized gold NPs were obtained using a UV-vis spectrophotometer (PerkinElmer). The morphology of the substrates following assembly of gold NPs was determined with a scanning electron microscope (SEM) at $25 \mathrm{kV}$ (Zeiss EVO LS10). The roughness of the substrate was measured with atomic force 
microscopy (AFM) from Nanomagnetics Instruments. A Witec alpha300 M+ model Raman spectrophotometer (50× objective) was used to observe the scattering by $532 \mathrm{~nm}$ wavelength laser $(2 \mu \mathrm{m}$ spot size). The reporter molecule, rhodamine $6 \mathrm{G}$, was drop-cast $(2$ $\mu \mathrm{L}, 100 \mu \mathrm{M}$ in ethanol), and the Raman scattering measurement was performed after drying of ethanol. The deposition of rhodamine $6 \mathrm{G}$ from the ethanolic solution resulted in complete wetting of the surface and spreading out to cover the whole substrate $\left(\sim 1 \mathrm{~cm}^{2}\right)$. Raman mappings $\left(40 \times 40 \mu \mathrm{m}^{2}\right)$ were generated based on the peak of rhodamine $6 \mathrm{G}$ at $1361 \mathrm{~cm}^{-1}$. Each mapping measurement included 6400 spectra obtained with an integration time of $0.005 \mathrm{~s}$. In the Raman measurements for the determination of the detection limit of the plasmonic substrate, a $2 \mu \mathrm{L}$ droplet of rhodamine $6 \mathrm{G}$ with a concentration that ranges from $10^{-5} \mathrm{M}$ to $10^{-10} \mathrm{M}$ was drop-cast on the green synthesized gold NPs assembled by repeating the immobilization 5 times, $1 \mathrm{~h}$ each. The integration time was set as 5 $\mathrm{s}$ for the determination of the detection limit and enhancement factor. The presented Raman scattering spectra and SERS intensities were obtained from mappings taken at three different regions of the substrate. The standard deviation in the intensity at $1361 \mathrm{~cm}^{-1}$ was calculated based on 1024 different spectra taken from a Raman mapping obtained over an area of $40 \times 40 \mu \mathrm{m}^{2}$. Attenuated total reflection-Fourier transform infrared (ATR-FTIR) spectra were obtained in the range of $500-4000 \mathrm{~cm}^{-1}$ using a PerkinElmer 400 FTIR spectrometer with a MIRacle ATR accessory. In ATR-FTIR analysis, an aliquot of the gold NPs and extract were first drop-cast on the cover glass and dried at $40{ }^{\circ} \mathrm{C}$ on a hot-plate. Transmission electron microscopy (TEM) analysis was performed using the FEITecnai G2 F30 instrument. For TEM characterization, the suspension of gold NPs was drop-cast on the 400-mesh copper grid and dried out at room temperature. Unless otherwise stated, at least three measurements were performed for each analysis. The reported values are arithmetic average of these measurements.

\section{RESULTS AND DISCUSSION}

All-Water Based Fabrication and Characterization of End-Grafted PEG Layers. Figure 1 schematically shows the important steps in the eco-friendly fabrication of plasmonically active solid substrates. A silicon wafer or glass substrate with surface silanol groups serves as the substrate. A thin film of PEG is then spin-coated on the substrate from an aqueous solution. A brief annealing facilitates the condensation reaction between the surface silanols and hydroxyl end-groups of PEG resulting in grafting of the polymer chains through the formation of a $\mathrm{Si}-\mathrm{O}-\mathrm{C}$ bond. ${ }^{29}$ Following removal of the excess material from the surface through washing in water, the substrate modified with end-grafted PEG layers is ready for use. Both the solvent and polymers can be safely disposed without the need for any postprocessing, since water and PEG are biocompatible. Plasmonic gold NPs can now be assembled on end-grafted PEG layers. Conventional citrate-stabilized NPs as well as gold NPs synthesized by the use of reducing agents derived from plants can be employed to decorate the PEGgrafted substrate. The resulting substrate exhibits high plasmonic activity and serves as a substrate in SERS.

The important steps that deserve attention from a green chemistry perspective in the fabrication of plasmonic structures on substrates modified with end-grafted PEG layers are the deposition of the polymer film, removal of the excess material following grafting, and synthesis of metallic NPs. The deposition of polymer thin films is commonly performed by spin-coating. Recent works by others and us have used organic solvents such as chlorobenzene and chloroform for spincoating PEG films. ${ }^{25,30,31}$ Chlorobenzene, in particular is a good solvent for spin-coating of uniform thin films $(<100 \mathrm{~nm})$ with broad usage in demanding nanolithography applica-

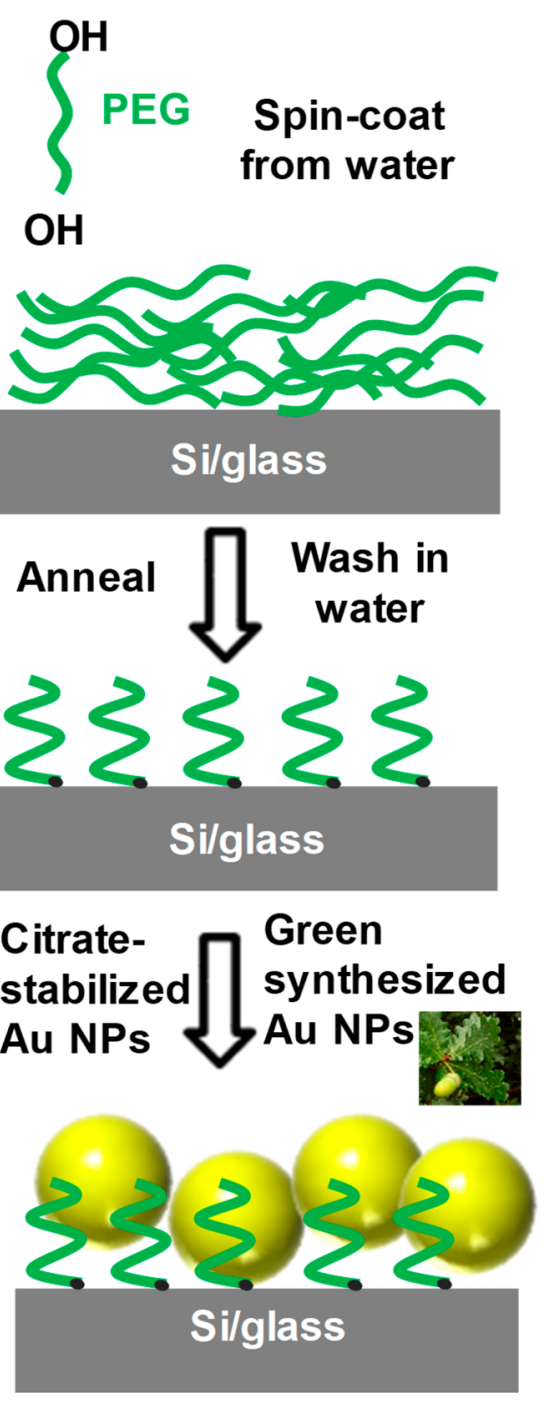

Figure 1. Environmentally benign approach for the fabrication of plasmonically active solid substrates.

tions; $^{32,33}$ however, it is a highly hazardous solvent. Following a thermal annealing step in a glovebox, the excess PEG has been typically removed by sonicating the substrate in chloroform, which readily dissolves PEG and is a volatile solvent that can be easily dried. The sequential washing steps result in significant amount of solvent usage. A substrate with a surface area of $1 \mathrm{~cm}^{2}$, for example, requires $\sim 60 \mathrm{~mL}$ of chloroform in three cycles of sonication. Replacing the organic solvents with a green solvent that does not require any special waste management greatly contributes to the sustainability of this process for practical applications. Finally, the use of plant extracts in the synthesis of gold NPs is investigated to minimize consumption of synthetic chemicals and benefit from waste biomaterials.

We first investigate the deposition of PEG films from aqueous solutions. Despite the high solubility of PEG in water, the high surface tension of water challenges spin-coating of uniform films. A key advantage of our grafting scheme is that only a fraction of the deposited polymers is grafted onto the substrate and the excess material is removed in the washing step. We, therefore, hypothesized that spin-coating thick films from water is important to cover the whole substrate with the polymer and compensate the nonuniformity of the films spin- 
Table 1. Thickness (nm) of Films after Spin-Coating and Washing Processes ${ }^{a}$

$\begin{array}{llllcc} & 2 \text { wt \% W } & 4 \text { wt \% W } & 6 \text { wt \% W } & 8 \text { wt \% W } & 2 \text { wt \% CB } \\ \text { spin-coat } & 41.5 \pm 4.8 & 95.7 \pm 3.2 & 160.2 \pm 3.4 & 270.7 \pm 3.5 & 365.5 \pm 3.7 \\ \text { wash } & 10.2 \pm 0.1 & 10.6 \pm 0.1 & 11.4 \pm 0.2 & 9.6 \pm 0.2 & 8.9 \pm 0.1\end{array}$

${ }^{a}$ Each column represents a different weight percentage of the polymer and solvent (W, water; CB, chlorobenzene) used in spin-coating.

coated from aqueous solutions. A series of films were spincoated on top of the freshly cleaned silicon substrates from water by varying the weight percentage of the dissolved PEG from 2 wt $\%$ to $10 \mathrm{wt} \%$. A reference sample was prepared by spin-coating from 2 wt \% PEG in chlorobenzene. Annealing these samples in a controlled environment at $180{ }^{\circ} \mathrm{C}$ for $5 \mathrm{~min}$ resulted in grafting of the polymer through the condensation reaction between the hydroxyl end-groups and surface silanols. ${ }^{34,35}$ Then, repeated sonication in chloroform removed the excess material. Table 1 lists the thicknesses of the spincoated films and end-grafted PEG layers, which were measured following the thermal annealing and washing steps, respectively. The thickness of the films spin-coated from water increased with the concentration of PEG in the solution. The films spin-coated from water were not as uniform as the reference sample using chlorobenzene as the solvent, and as a result the standard deviations in the thickness values were higher. Following thermal annealing and washing of the excess film, the thickness of end-grafted polymers prepared by spincoating from aqueous solutions ranged between 8.9 and 11.4 $\mathrm{nm}$, whereas the reference sample had a thickness of $12.2 \mathrm{~nm}$. The highest thickness was obtained in the case of films spincoated from 6 wt \% solution. These results suggest the possibility of achieving grafting densities $\left(\sim 0.21\right.$ chains $/ \mathrm{nm}^{2}$, see the Supporting Information for details) that are comparable with the reference sample using all-water based deposition of the PEG films. The distance between the grafting sites (see the Supporting Information for details) is smaller than the $2 R_{\mathrm{g}}$ (radius of gyration), suggesting that end-grafted PEG layers are in the brush regime. ${ }^{36}$ Despite nonuniformities of the initial films that were spin-coated from water, highly uniform layers of end-grafted polymers were obtained at the end of the process. The roughness of the end-grafted PEG layers deposited from water was $\sim 1.1 \mathrm{~nm}$, which is on par with the $0.9 \mathrm{~nm}$ roughness obtained with the reference sample prepared by spin-coating from chlorobenzene (Figure 2a,b). The surface of the end-grafted PEG layers exhibited domain like structures that are likely a result of the crystals formed by the PEG chains. ${ }^{37}$

The functionality of end-grafted PEG layers prepared by deposition from aqueous solutions was determined by ex-situ SEM imaging and SERS measurements. Citrate-stabilized spherical gold NPs with a diameter of $20 \mathrm{~nm}$ were immobilized on the grafted PEG layers. The substrates were washed in water under sonication to remove weakly bound particles from

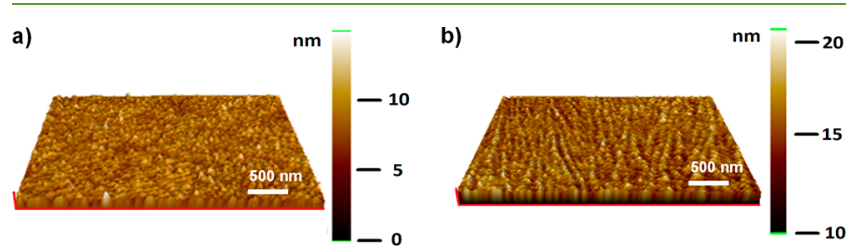

Figure 2. AFM images of end-grafted PEG layers following thermal annealing and washing of the films spin-coated from (a) 2 wt \% PEG in chlorobenzene and (b) 6 wt \% PEG in water.
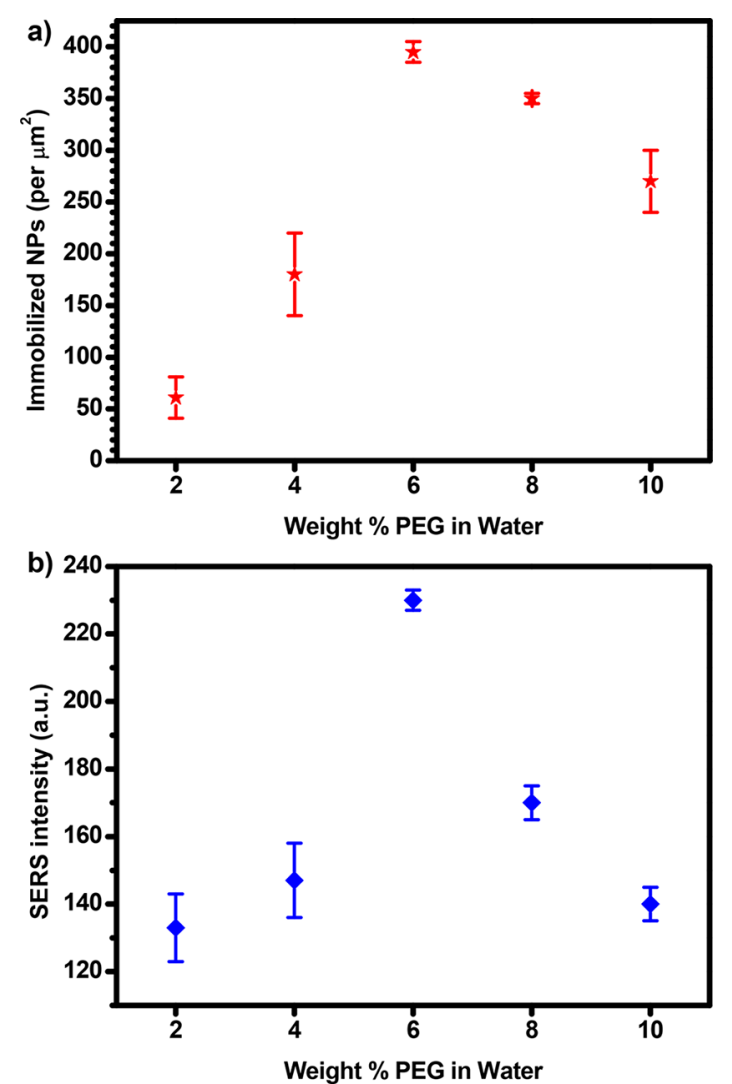

Figure 3. Effect of the composition of the spin-coating solution on the density of the immobilized gold NPs and their SERS response. (a) Variation of the surface density of immobilized NPs $(20 \mathrm{~nm}$ in diameter) as a function of the weight percentage of PEG in the aqueous spin-coating solution. (b) SERS intensity at a position of $1361 \mathrm{~cm}^{-1}$ for rhodamine $6 \mathrm{G}$ deposited from an ethanolic solution at a concentration of $100 \mu \mathrm{M}$ on the assembled NPs given in part a. Citrate-stabilized $\mathrm{Au}$ NPs were assembled by immobilization for $1 \mathrm{~h}$ followed by washing in water.

the surface. Figure $3 a$ presents the average number of the immobilized gold NPs derived from SEM images. The binding of the gold NPs exhibited a variation as a function of the weight percentage of PEG in aqueous solutions used to spincoat the films. The average surface density of the particles was the highest $\left(390 \pm 9 \mathrm{NPs} / \mu \mathrm{m}^{2}\right)$ in the case of films spincoated from aqueous solutions containing 6 wt \% PEG. For 2 and 4 wt $\%$ solutions, the density of immobilized particles was remarkably low and the particles assembled into aggregates. SERS measurements provide an indirect information about the plasmonic activity of the substrates, since the enhancement of signals in Raman spectroscopy is directly proportional to the density of immobilized NPs, which leads to electric field magnification through the excitation of surface plasmon resonances. Figure $3 \mathrm{~b}$ shows the intensity of the characteristic peak of the reporter molecule for PEG films spin-coated from solutions of varying composition. In parallel to the density of the immobilized particles, the intensity of the SERS signal was 
the highest in the case of end-grafted PEG layers prepared by films spin-coated from 6 wt $\%$ aqueous solution. The intensity of the signal was $\sim 1.5$-fold higher than that of the reference sample prepared by using chlorobenzene. Despite the lower number of the immobilized gold nanoparticles for the 4 wt \% in comparison to the $10 \mathrm{wt} \%$, the SERS intensity is higher possibly due to the formation of plasmonic hot-spots in the gap regions of close-packed assemblies (see Figure S1) that are specifically observed for this concentration of PEG. As evidenced from the large error bar in the SERS intensity for the 4 wt \%, such hot-spots are not uniformly distributed over the entire substrate. It is likely that at the low concentrations ( 2 and 4 wt \%) of PEG, nonuniformities in the spin-coated films resulted in end-grafted layers with a certain degree of inhomogeneity, evidenced with microscopic variations in the density of bound NPs (see Figure S1a). At high concentrations (e.g., $10 \mathrm{wt} \%$ ) of PEG, water trapped in the thick films may have a negative impact on the grafting process leading to reductions in the thickness of the grafted layer, density of bound NPs, and SERS intensity. Excess water molecules in the film may hinder driving of the condensation reaction between the end-hydroxyl group of the polymer and surface silanol of the substrate to the grafting side because water is a product of this reaction. Therefore, we chose 6 wt \% PEG as the solution for deposition of PEG films for the rest of the study based on the thickness of grafted layers, density of immobilized particles, and SERS measurements.

Second, we investigated the washing step used for removal of the excess and unreacted PEG from the substrate. The films were prepared by spin-coating of solutions containing 6 and 2 wt \% PEG in water and chlorobenzene, respectively. The films were then thermally annealed to graft the polymer to the substrate. The subsequent washing step was performed either in water or chloroform. The thickness of the end-grafted PEG layers washed in chloroform was slightly higher than those washed in water (see Table S1). This minor contrast in the thickness may arise from the difference in the conformation of PEG molecules in these solvents. ${ }^{38}$ The density of immobilized gold NPs and their SERS activity were similar for both washing solvents (see Table S1 and Figure S2). These results suggest that the high solubility of PEG in both solvents is sufficient for the effective removal of the polymer. Water is relatively more difficult to dry than chloroform, and it is likely that surface bound water molecules may remain attached to the surface without an additional heat treatment. Such bound water molecules are not expected to have any adverse effects, since the subsequent immobilization step is performed in an aqueous solution.

Size-Dependent Assembly of Citrate-Stabilized Gold NPs. All-water based fabrication of end-grafted PEG layers enables size-dependent assembly of citrate-stabilized gold NPs with a density that is better than those achieved with organic solvents. Previous work has revealed the unique behavior of substrates functionalized with end-grafted PEG layers: the binding density (the number of the immobilized NPs per unit area) and surface coverage (the fraction of the substrate surface that is covered with the immobilized NPs) of the citratestabilized gold NPs increase with decreasing particle diameter. $^{24,30}$ Figure $4 \mathrm{a}$ shows that all-water based processing of end-grafted PEG layers led to similar behavior: the density of the immobilized gold NPs decreased with increasing size of the particles (see Figure S3 for details). We note that the density of the immobilized particles was consistently higher on
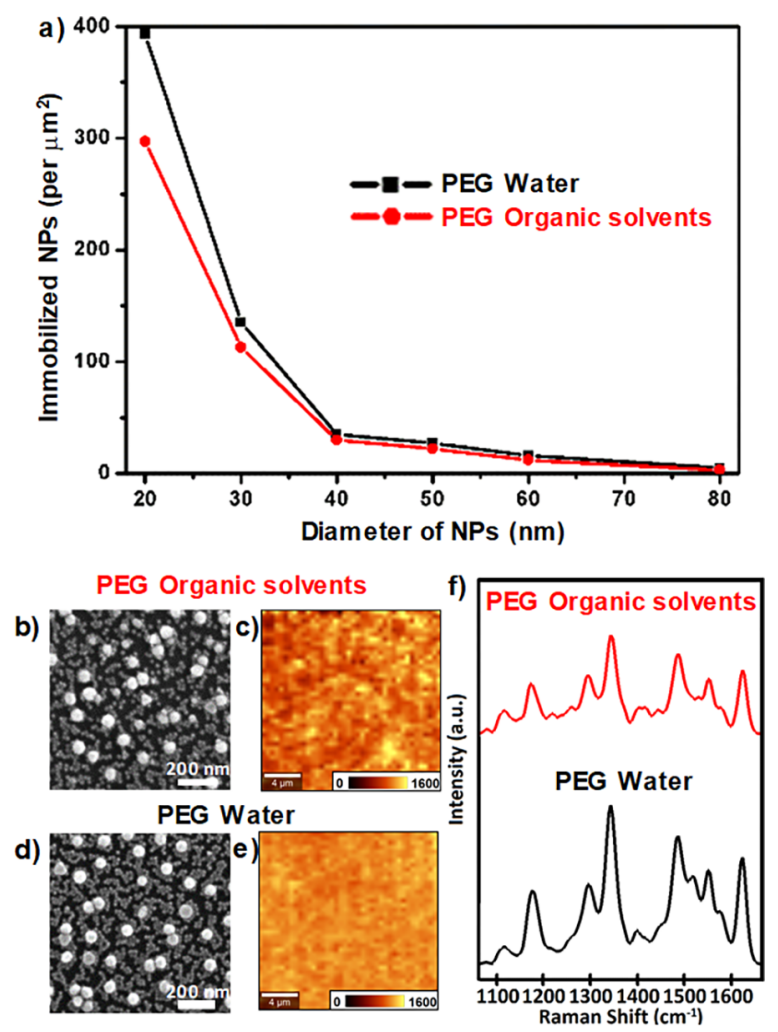

Figure 4. Size dependent assembly of citrate-stabilized gold NPs on PEG grafted substrates. (a) Surface density of gold NPs immobilized on end-grafted PEG layers as a function of the diameter of the particles. Citrate-stabilized Au NPs were assembled by immobilization for $1 \mathrm{~h}$ followed by washing in water. (b,d) SEM images and (c,e) Raman mappings (based on the SERS intensity at a position of 1361 $\left.\mathrm{cm}^{-1}\right)$ of heterostructures of gold NPs $(60$ and $20 \mathrm{~nm})$ assembled on end-grafted PEG layers. (f) SERS spectra of the reporter molecule, which consists of rhodamine $6 \mathrm{G}$ deposited from an ethanolic solution at a concentration of $100 \mu \mathrm{M}$. The results are given for end-grafted PEG layers that were prepared by all-water and organic solvent-based processing. Heterostructures of gold NPs were assembled by repeating the immobilization process $(1 \mathrm{~h}$ treatment and $2 \mathrm{~min}$ wash in water under sonication) for 5 times.

PEG grafted substrates processed by water in comparison with those prepared with organic solvents. The difference in the density was more profound for the small particles (e.g., $20 \mathrm{~nm}$ ) than the large ones. We hypothesize that this difference is related to the crystallinity of PEG induced by water-based processing. The decrease of the binding density of the citratestabilized gold NPs with increasing particle size becomes an advantage in assembly of plasmonic heterostructures, which are clusters of particles that differ in size. ${ }^{39}$ The close placement of NPs of varying size results in asymmetric resonances and extremely high levels of electromagnetic field enhancement in the gap regions. ${ }^{22}$ A recent study ${ }^{40}$ has shown that the strength of the field enhancement and SERS intensities are extremely high in the gap regions in between the two particles of varying size (e.g., 60 and $30 \mathrm{~nm}$ ). Heterostructures of citrate-stabilized gold NPs were assembled on the end-grafted PEG layers by sequential deposition of particles with diameters of 60 and 20 $\mathrm{nm}$. The diameters of NPs were chosen based on the sufficient contrast in the binding density of the particles on end-grafted PEG layers. To maximize the surface coverage of both sizes of the particles, the immobilization process was repeated for 5 times. ${ }^{20}$ The heterostructures were effectively assembled over 
large areas (Figure 4). The intensity of the characteristic peak of the reporter molecule on the heterostructures prepared by water-based processing was stronger than the organic solvent based one. This result likely relates to the higher density of immobilized particles on the former type of the substrate.

Green Synthesis and Assembly of Gold NPs. Synthesis of metallic NPs with green chemistry approaches has recently gained significant interest for a broad range of applications. ${ }^{41}$ Herein we investigate assembly of gold NPs synthesized via a green chemistry approach on PEG grafted substrates prepared by all water-based processing. We used the extract of Quercus pubescens as a reducing and stabilizing agent in the synthesis of gold NPs. ${ }^{42,43}$ The synthesis of the gold NPs was performed by varying concentrations of the gold precursor $\left(\mathrm{HAuCl}_{4}\right)$, amounts of the extract and reaction times. When $1 \mathrm{mM}$ gold precursor was used, the synthesis led to a mixture of prisms and spherical particles as confirmed with TEM images (see Figure S4). The spherical particles and the prisms exhibited surface plasmon resonance peaks around 540 and $900 \mathrm{~nm}$ in UV-vis spectra, respectively (see Figure S5). Different chemical species found in the plant extract likely contribute to multiple nucleation and growth processes challenging the synthesis of monodisperse particles. The size and shape inhomogeneity are known for reducing agents that have additional functions such as a complexant. ${ }^{44}$ The growth of the prisms could be minimized when the concentration of the gold precursor was reduced to $0.5 \mathrm{mM}$. Increasing the volume of the extract at $0.5 \mathrm{mM} \mathrm{HAuCl}_{4}$ resulted in mostly spherical NPs with a single surface plasmon resonance peak at $\sim 535 \mathrm{~nm}$ with an average diameter of $25.6 \pm 11.1 \mathrm{~nm}$ (Figure 5). Further
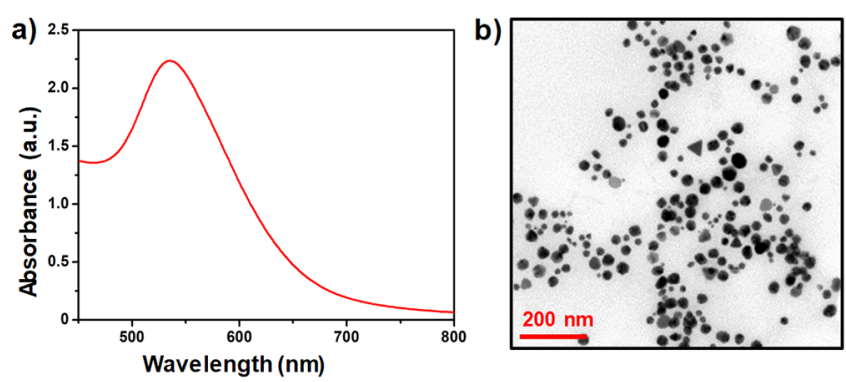

c)

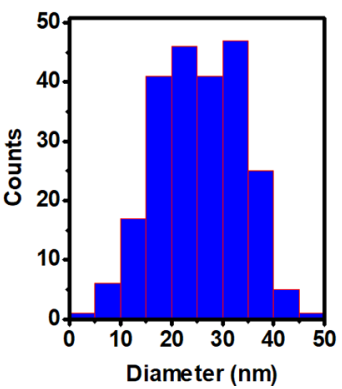

d)

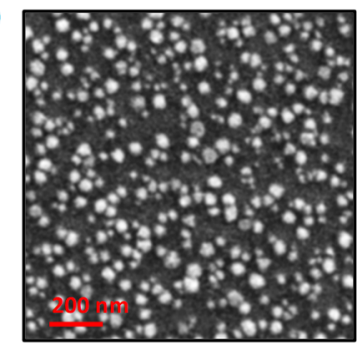

Figure 5. Gold NPs synthesized via a green chemistry approach using the extract derived from Quercus pubescens: (a) UV-vis spectra of the gold NPs, (b) TEM image of the gold NPs, (c) distribution of the diameter of the particles obtained from TEM images, and (d) SEM images of the assembled gold NPs on end-grafted PEG layers prepared by all-water based processing. Green synthesized gold NPs were assembled by repeating the immobilization process $(1 \mathrm{~h}$ treatment and 2 min wash in water under sonication) for 5 times. Gold NPs were synthesized using $0.5 \mathrm{mM} \mathrm{HAuCl} 4,0.75 \mathrm{~mL}$ extract, and $5 \mathrm{~min}$ of reaction time. reduction of the gold precursor concentration to $0.1 \mathrm{mM}$ did not lead to a distinguishable growth of the particles for different reaction times and amounts of the extract as shown via UV-vis spectra (see Figure S5 for details). The immobilization of these green synthesized NPs on silicon substrates modified with end-grafted PEG layers resulted in the assembly of the particles with a surface coverage that is on par with the citrate-stabilized particles (Figure 5d). To the best of our knowledge, this is the first demonstration of assembly of gold NPs stabilized with a reducing agent other than citrate on end-grafted PEG layers. The ability to immobilize the green synthesized gold NPs on end-grafted PEG layers suggests that the ligands are weakly associated with the surface of the particle, since PEG chains are unable to bind the gold NPs, which were stabilized with covalently bound ligands. ${ }^{24}$ A very recent work ${ }^{45}$ has shown that gold nanorods stabilized with proper ligands can be selectively immobilized on end-grafted PEG layers. The green synthesis of gold NPs with different shapes and their assembly on end-grafted PEG layers that were prepared by all-water based processing can enable ecofriendly fabrication of complex plasmonic nanostructures.

To characterize the chemical structure, we performed infrared spectroscopy analysis of the extract and the green synthesized gold NPs. Both the extract and green synthesized gold NPs exhibited prominent peaks that correspond to phenolic compounds and fatty acids (see Figure S6 for details) in close agreement with the literature. ${ }^{46,47}$ The phenolic hydroxyl groups and carboxylic acids have been reported for their roles as reducing and stabilizing agents in the phytosynthesis of NPs. ${ }^{41}$ In the case of the citrate-stabilized gold NPs, carboxylate groups play a critical role in the adsorption of the citrate anions on the surface of the particle. ${ }^{48}$ Such carboxylate groups are evident in the spectra of both the trisodium citrate and citrate-stabilized gold NPs. The presence of similar peaks in the case of the green synthesized gold NPs suggests that similar groups contribute to the stabilization of gold NPs and their interaction with the end-grafted PEG layers.

SERS Performance. Figure 6 presents the SERS capabilities of the plasmonically active substrates fabricated by assembly of green synthesized gold NPs on end-grafted PEG layers prepared by all-water based processing. High levels of uniformity in grafting of PEG and assembly of gold NPs resulted in the ability to receive high SERS intensities over the entire substrate. Figure 6a reveals the variation in the intensity of the characteristic peak of rhodamine $6 \mathrm{G}$ at $1361 \mathrm{~cm}^{-1}$ associated with the aromatic $\mathrm{C}-\mathrm{C}$ stretching vibrations ${ }^{49}$ obtained using 1024 different spectra taken from a Raman mapping with an area of $40 \times 40 \mu \mathrm{m}^{2}$. From the collected data, the average intensity was found to be 8821.5 counts resulting in an analytical enhancement factor ${ }^{50}$ of $9.48 \times 10^{4}$ (see Supporting Information for details of the calculation) using the Raman peak intensity at $1361 \mathrm{~cm}^{-1}$ for rhodamine $6 \mathrm{G}$ under $532 \mathrm{~nm}$ laser excitation. The standard deviation in the average intensity obtained over 1024 different spectra was $28.8 \%$. The Raman mapping presented in Figure $6 \mathrm{~b}$ shows that the SERS signals could be received from the entire substrate confirming that the substrate was successfully functionalized with endgrafted PEG layers in our all-water based fabrication process yielding in dense assembly of green synthesized gold NPs with high plasmonic activity (see Figure S7 to see the results with citrate-stabilized gold NPs). To determine the level of concentration that the signal can be detected from the fabricated plasmonic substrate, we deposited rhodamine $6 \mathrm{G}$ 
a)
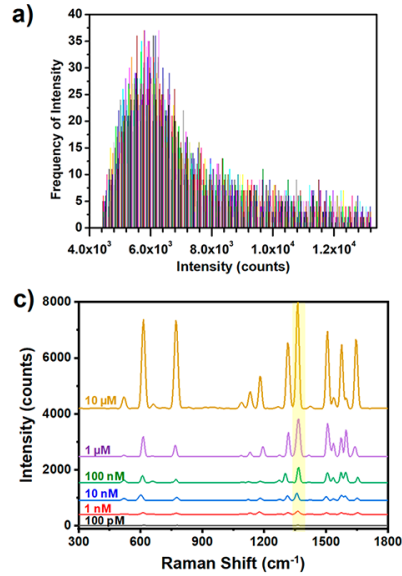

b)
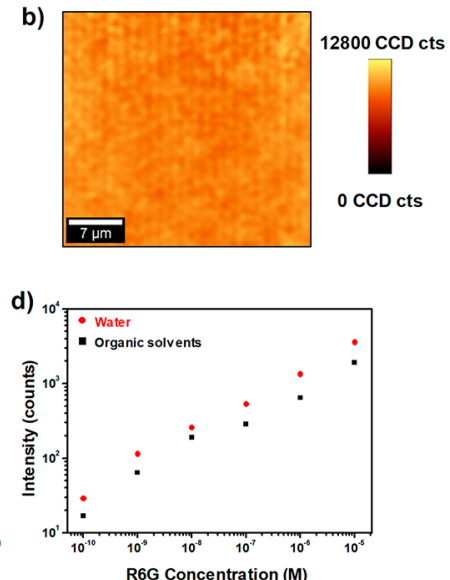

Figure 6. SERS performance of the green synthesized gold NPs assembled on PEG grafted substrates fabricated by all-water based processing. (a) Raman scattering intensity at a position of $1361 \mathrm{~cm}^{-1}$ for rhodamine 6G obtained using 1024 different spectra taken from (b) Raman mapping with an area of $40 \times 40 \mu \mathrm{m}^{2}$. The concentration of rhodamine $6 \mathrm{G}$ is $100 \mu \mathrm{M}$. (c) Raman spectra of varying concentrations of rhodamine $6 \mathrm{G}$ on the plasmonically active substrate. The concentration of rhodamine $6 \mathrm{G}$ is given in the plot. (d) Intensity at $1361 \mathrm{~cm}^{-1}$ as a function of the concentration of rhodamine $6 \mathrm{G}$ on a logarithmic scale for the substrates prepared by all-water and organic solvent-based processing of end-grafted PEG layers followed by assembly of the green synthesized gold NPs. Gold NPs were assembled by repeating the immobilization process $(1 \mathrm{~h}$ treatment and $2 \mathrm{~min}$ wash in water under sonication) for 5 times.

from solutions of different concentrations $\left(10^{-5}-10^{-10} \mathrm{M}\right) .^{51}$ The characteristic bands of the reporter molecule could be clearly detected at the concentration as low as $100 \mathrm{pM}$ (Figure $6 c)$. Note that at concentrations that are lower than $10 \mathrm{nM}$, the
SERS intensity exhibited a certain degree of variation over the substrate (see Figure S8). The SERS response of the assembled green synthesized gold NPs was higher for the entire range of the concentrations on the end-grafted PEG layers prepared by water-based processing in comparison with those prepared using organic solvents (Figure $6 \mathrm{~d}$ ). This contrast is likely a result of the higher surface coverage of the particles and reduced interparticle distances obtained on the end-grafted PEG layers processed in water. Previous studies have shown that the localization of strong electric fields near the small gaps in between the metallic NPs enhances the Raman scattering from the substrate. ${ }^{52}$ The Raman intensity of the characteristic peak increased with the concentration of the reporter molecule deposited on the plasmonically active substrate (Figure 6d). The relationship between the SERS intensity $(I)$ and concentration $(C)$ of rhodamine $6 \mathrm{G}$ can be well fitted using the expression of $\log (C)=5.5537+0.3995 \times \log (I)$ with a coefficient of determination $\left(R^{2}\right)$ of 0.9913 . This relationship suggests the promise of this platform for quantitative detection of molecules in SERS. Together with the unique advantages in terms of the sustainability, scalability, simplicity, and robustness, the present study compares well with the recently reported SERS substrates. ${ }^{53-57}$

Sensing Application. Finally, we demonstrate the use of the environmentally benign plasmonics platform in the construction of a SERS based sensor for detection of multiple molecules. For this purpose, we prepared plasmonically active substrates using gold NPs synthesized via the extract of Quercus pubescens (Figure 7) and heterostructures that consist of citrate-stabilized gold NPs (see supporting Figure S9) on end-grafted PEG layers prepared by all-water based processing. The delivery of multiple analyte molecules to spatially defined regions was accomplished by using an elastomeric mold containing microfluidic channels that are connected to the a)

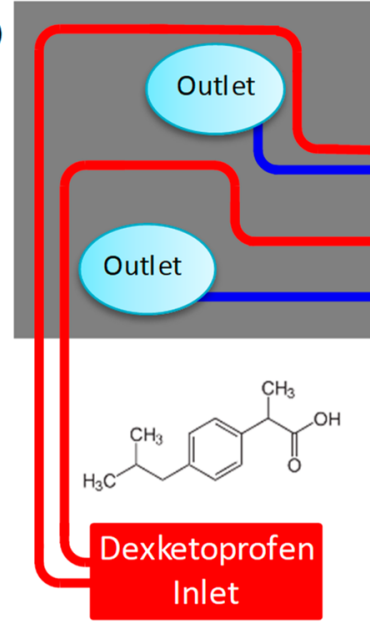

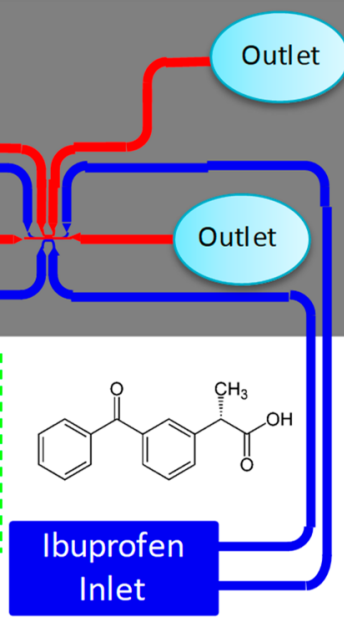
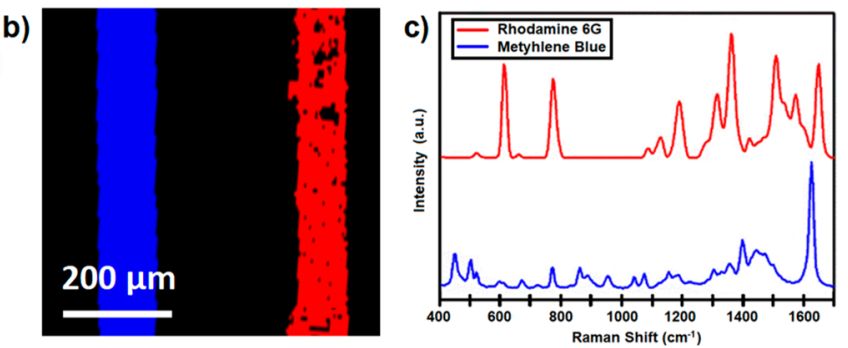

d)

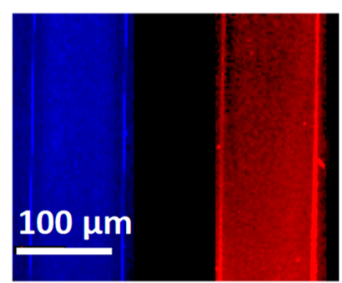

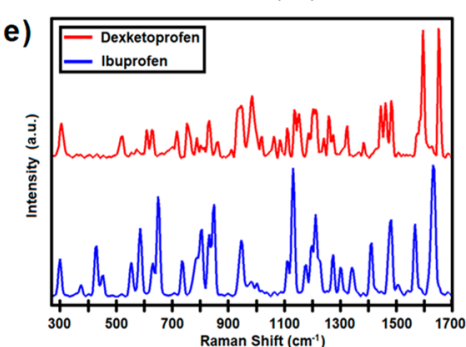

Figure 7. Sensing of multiple molecules on the environmentally benign plasmonic substrate: (a) layout of the microfluidic channels and (b) SERS mapping image of methylene blue $(100 \mu \mathrm{M})$ and rhodamine $6 \mathrm{G}(100 \mu \mathrm{M})$ deposited on the plasmonic substrate consisting of the green synthesized gold NPs assembled on all-water processed end-grafted PEG layers. Gold NPs were assembled by repeating the immobilization process ( $1 \mathrm{~h}$ treatment and $2 \mathrm{~min}$ wash in water under sonication) for 5 times. The mapping image was generated by using the characteristic peaks of rhodamine $6 \mathrm{G}$ and methylene blue at positions of 1361 and $1624 \mathrm{~cm}^{-1}$, respectively. (c) Raman spectra of rhodamine 6G and methylene blue and (d) SERS mapping image of dexketoprofen $(3.9 \mathrm{mM})$ and ibuprofen $(4.8 \mathrm{mM})$ on the plasmonic substrate. The mapping image was generated by using the characteristic peaks of dexketoprofen and ibuprofen at positions of 1596 and $1633 \mathrm{~cm}^{-1}$, respectively. (e) Raman spectra of dexketoprofen and ibuprofen. The delivery of the drug and reporter molecules through the microfluidic channels was performed using solutions prepared in ethanol. 
reservoir (Figure 7a). Placing the mold over the substrate with assembled arrays of gold NPs, resulted in open-ended continuous channels over a length of $1 \mathrm{~cm}$. The controlled application of a vacuum at the outlet of the channel resulted in transport of the analyte solution from the inlet to the outlet at an average velocity of $0.5 \mathrm{~mm} / \mathrm{s}$. Following the filling of the channels and evaporation of the solvent, the mold was removed from the substrate. The patterned deposition of multiple analyte molecules allowed for simultaneous analysis and direct comparison of different samples. Two commonly employed SERS reporter molecules, rhodamine 6G and methylene blue, were deposited on the NP arrays. Both molecules could be detected on the same substrate surface (Figure $7 \mathrm{~b}, \mathrm{c}$ ). The plasmonic gold NPs effectively increased the inelastic scattering from both rhodamine $6 \mathrm{G}$ and methylene blue, enabling detection of the molecules via Raman spectroscopy (see Figure S10a). Mapping the characteristic peaks of rhodamine $6 \mathrm{G}$ and methylene blue confirmed the presence of the molecules in the alternating channels. The microfluidics integrated SERS platform can successfully pattern and detect analyte molecules at concentrations as low as $100 \mathrm{nM}$. A further reduction in the concentration results in nonuniform deposition of the molecules within the channels (see Figure S11). The intense signals in SERS as well as the ability to pattern multiple molecules within microfluidic channels may be well utilized in fabrication of security labels, ${ }^{58}$ sensing of gaseous molecules, ${ }^{59}$ and multiplexed suspension assays. ${ }^{60}$

To demonstrate the practical value of the microfluidics integrated SERS platform, we investigated detection of two types of nonsteroidal anti-inflammatory drugs (NSAIDs), which are commonly consumed by the public and present as contaminants in the environment. NSAIDs are effective pain relievers that primarily function by the inhibition of cyclooxygenase enzymes. ${ }^{61}$ These drugs can come in contact with wastewater streams through ingestion followed by excretion and direct disposal of unused drugs. ${ }^{62}$ Studies have demonstrated incomplete degradability of such pharmaceuticals in some wastewater treatment plants. ${ }^{63,64}$ Even at reduced concentrations, long-term exposure to such drugs has potential to facilitate alterations in the ecosystem through toxicity and generation of resistance to these drugs. Therefore, it is of great interest to be able to simultaneously detect multiple drug molecules at low concentrations. Herein we investigate simultaneous detection of ibuprofen and dexketoprofen through the microfluidics integrated environmentally benign SERS platform. Both ibuprofen and dexketoprofen belong to the propionic acids family of NSAIDs and are widely used for pain management. ${ }^{65}$ For these experiments, the elastomeric mold was conformally contacted over the plasmonic substrate and solutions of the drugs were sucked into the channels as described above. Following detachment of the mold from the surface, the characteristic Raman fingerprints of ibuprofen and dexketoprofen were obtained over the consecutive channels (Figure $7 \mathrm{~d}, \mathrm{e}$ ). Note that there were no detectable signals on the bare silicon substrate in the absence of plasmonic gold NPs (see Figure S10b). Mapping the intensity of the Raman peaks of ibuprofen and dexketoprofen at 1633 and $1596 \mathrm{~cm}^{-1}$ clearly showed the simultaneous detection of the multiple drug molecules.

\section{CONCLUSIONS}

In summary, fabrication of plasmonically active solid substrates with the use of biocompatible materials and solely water-based solvents was demonstrated. Highly smooth surfaces consisting of end-grafted PEG layers were obtained via one-step facile grafting of an industrially available end-functional polymer. The substrates modified with end-grafted PEG layers resulted in uniformly and strongly bound gold NP arrays, which exhibit high plasmonic activity significantly enhancing the intensity of signals in Raman spectroscopy. The assembly of gold NPs synthesized by the use of plant extracts on the end-grafted PEG layers not only contributed to the sustainability of the process but also demonstrated the interaction between endgrafted PEG layers and gold NPs stabilized with a reducing agent other than citrate for the first time. Simultaneous SERS sensing of multiple drug molecules with a microfluidic device presents an example to the potential use of the presented platform. The sustainable, scalable, simple, and robust fabrication of plasmonically active solid substrates is expected to have a broad impact in a range of fields including catalysis, anticounterfeiting technologies, sensing, and metasurfaces.

\section{ASSOCIATED CONTENT}

\section{Supporting Information}

The Supporting Information is available free of charge on the ACS Publications website at DOI: 10.1021/acssuschemeng.8b06133.

Calculation of grafting density and enhancement factor, additional SEM images and Raman spectra on the effect of the spin-coating and washing solvent, TEM images, UV-vis spectra and FTIR spectra of green NPs, and SERS performance and sensing on heterostructures of gold NPs (PDF)

\section{AUTHOR INFORMATION}

\section{Corresponding Authors}

*E-mail: onses@erciyes.edu.tr.

*E-mail: elbuken@unam.bilkent.edu.tr.

*E-mail: fduman@erciyes.edu.tr.

*E-mail: nanobiotechnology@gmail.com.

ORCID

Caglar Elbuken: 0000-0001-8359-6871

Gokhan Demirel: 0000-0002-9778-917X

M. Serdar Onses: 0000-0001-6898-7700

\section{Notes}

The authors declare no competing financial interest.

\section{ACKNOWLEDGMENTS}

This work was supported by the Research Fund of the Erciyes University (Project Number FBA-2016-6711). M.S.O. and G.D. acknowledge partial support from the Turkish Academy of Sciences Distinguished Young Scientist Award (TUBAGEBIP).

\section{REFERENCES}

(1) Karker, N.; Dharmalingam, G.; Carpenter, M. A. Thermal energy harvesting plasmonic based chemical sensors. ACS Nano 2014, 8 (10), 10953-10962.

(2) Xiao, C.; Hu, H.; Zhang, X.; MacFarlane, D. R. Nanostructured gold/bismutite hybrid heterocatalysts for plasmon-enhanced photo- 
synthesis of ammonia. ACS Sustainable Chem. Eng. 2017, 5 (11), 10858-10863.

(3) Thakur, N. S.; Bhaumik, J.; Kirar, S.; Banerjee, U. C. Development of gold-based phototheranostic nanoagents through a bioinspired route and their applications in photodynamic therapy. ACS Sustainable Chem. Eng. 2017, 5 (9), 7950-7960.

(4) Reineck, P.; Lee, G. P.; Brick, D.; Karg, M.; Mulvaney, P.; Bach, U. A solid-state plasmonic solar cell via metal nanoparticle selfassembly. Adv. Mater. 2012, 24 (35), 4750-4755.

(5) Liang, Z.; Sun, J.; Jiang, Y.; Jiang, L.; Chen, X. Plasmonic enhanced optoelectronic devices. Plasmonics 2014, 9 (4), 859-866.

(6) Liz-Marzán, L. M.; Murphy, C. J.; Wang, J. Nanoplasmonics. Chem. Soc. Rev. 2014, 43 (11), 3820-3822.

(7) Zhang, C.; Zhao, H. Q.; Zhou, L. A.; Schlather, A. E.; Dong, L. L.; McClain, M. J.; Swearer, D. F.; Nordlander, P.; Halas, N. J. Al-Pd nanodisk heterodimers as antenna-reactor photocatalysts. Nano Lett. 2016, 16 (10), 6677-6682.

(8) Liu, N.; Tang, M. L.; Hentschel, M.; Giessen, H.; Alivisatos, A. P. Nanoantenna-enhanced gas sensing in a single tailored nanofocus. Nat. Mater. 2011, 10 (8), 631-636.

(9) Yang, P.; Zheng, J.; Xu, Y.; Zhang, Q.; Jiang, L. Colloidal synthesis and applications of plasmonic metal nanoparticles. Adv. Mater. 2016, 28 (47), 10508-10517.

(10) Metz, K. M.; Sanders, S. E.; Pender, J. P.; Dix, M. R.; Hinds, D. T.; Quinn, S. J.; Ward, A. D.; Duffy, P.; Cullen, R. J.; Colavita, P. E. Green synthesis of metal nanoparticles via natural extracts: the biogenic nanoparticle corona and its effects on reactivity. ACS Sustainable Chem. Eng. 2015, 3 (7), 1610-1617.

(11) Lee, S. Y.; Krishnamurthy, S.; Cho, C.-W.; Yun, Y.-S. Biosynthesis of gold nanoparticles using Ocimum sanctum extracts by solvents with different polarity. ACS Sustainable Chem. Eng. 2016, 4, 2651-2659.

(12) Raveendran, P.; Fu, J.; Wallen, S. L. Completely "green" synthesis and stabilization of metal nanoparticles. J. Am. Chem. Soc. 2003, 125 (46), 13940-13941.

(13) Çolak, H.; Karaköse, E.; Duman, F. High optoelectronic and antimicrobial performances of green synthesized $\mathrm{ZnO}$ nanoparticles using Aesculus hippocastanum. Environ. Chem. Lett. 2017, 15, 547552.

(14) Yilmaz, H.; Pekdemir, S.; Ipekci, H. H.; Kiremitler, N. B.; Hancer, M.; Onses, M. S. Ambient, rapid and facile deposition of polymer brushes for immobilization of plasmonic nanoparticles. Appl. Surf. Sci. 2016, 385, 299-307.

(15) Ben Haddada, M.; Hübner, M.; Casale, S.; Knopp, D.; Niessner, R.; Salmain, M.; Boujday, S. Gold nanoparticles assembly on silicon and gold surfaces: mechanism, stability and efficiency in diclofenac biosensing. J. Phys. Chem. C 2016, 120, 29302-29311.

(16) Onses, M. S.; Wan, L.; Liu, X. Y.; Kiremitler, N. B.; Yilmaz, H.; Nealey, P. F. Self-assembled nanoparticle arrays on chemical nanopatterns prepared using block copolymer lithography. ACS Macro Lett. 2015, 4 (12), 1356-1361.

(17) Harris, J. M. Introduction to Biotechnical and Biomedical Applications of Poly(Ethylene Glycol). In Poly(ethylene Glycol) Chemistry; Topics in Applied Chemistry; Springer: Boston, MA, 2013; pp 1-14,

(18) Fiume, M. M.; Heldreth, B.; Bergfeld, W. F.; Belsito, D. V.; Hill, R. A.; Klaassen, C. D.; Liebler, D.; Marks, J. G., Jr; Shank, R. C.; Slaga, T. J. Safety assessment of alkyl PEG ethers as used in cosmetics. Int. J. Toxicol. 2012, 31, 169S-244S.

(19) Hoffman, A. S. Hydrogels for biomedical applications. Adv. Drug Delivery Rev. 2012, 64, 18-23.

(20) Pekdemir, S.; Karabel, S.; Kiremitler, N. B.; Liu, X.; Nealey, P.; Onses, M. S. Modulating the kinetics of nanoparticle adsorption for simple and high yield fabrication of plasmonic heterostructures as SERS substrates. ChemPhysChem 2017, 18, 2114-2122.

(21) Meyerbroker, N.; Kriesche, T.; Zharnikov, M. Novel ultrathin poly(ethylene glycol) films as flexible platform for biological applications and plasmonics. ACS Appl. Mater. Interfaces 2013, 5 (7), 2641-2649.
(22) Liu, X. Y.; Biswas, S.; Jarrett, J. W.; Poutrina, E.; Urbas, A.; Knappenberger, K. L.; Vaia, R. A.; Nealey, P. F. Deterministic construction of plasmonic heterostructures in well-organized arrays for nanophotonic materials. Adv. Mater. 2015, 27 (45), 7314-7319.

(23) Kiremitler, N. B.; Pekdemir, S.; Patarroyo, J.; Karabel, S.; Torun, I.; Puntes, V. F.; Onses, M. S. Assembly of plasmonic nanoparticles on nanopatterns of polymer brushes fabricated by electrospin nanolithography. ACS Macro Lett. 2017, 6, 603-608.

(24) Diamanti, S.; Arifuzzaman, S.; Genzer, J.; Vaia, R. A. Tuning gold nanoparticle-poly(2-hydroxyethyl methacrylate) brush interactions: from reversible swelling to capture and release. ACS Nano 2009, 3 (4), 807-818.

(25) Meyerbroker, N.; Zharnikov, M. Hydrogel nanomembranes as templates for patterned deposition of nanoparticles on arbitrary substrates. ACS Appl. Mater. Interfaces 2014, 6 (16), 14729-14735.

(26) Wang, Y.; Yan, B.; Chen, L. SERS Tags: Novel Optical Nanoprobes for Bioanalysis. Chem. Rev. 2013, 113 (3), 1391-1428.

(27) Duffy, D. C.; McDonald, J. C.; Schueller, O. J. A.; Whitesides, G. M. Rapid prototyping of microfluidic systems in poly(dimethylsiloxane). Anal. Chem. 1998, 70 (23), 4974-4984.

(28) Xia, Y. N.; Whitesides, G. M. Soft lithography. Angew. Chem., Int. Ed. 1998, 37 (5), 550-575.

(29) Alcantar, N. A.; Aydil, E. S.; Israelachvili, J. N. Polyethylene Glycol-Coated Biocompatible Surfaces. J. Biomed. Mater. Res. 2000, 51 (3), 343-351.

(30) Onses, M. S.; Nealey, P. F. Tunable assembly of gold nanoparticles on nanopatterned poly(ethylene glycol) brushes. Small 2013, 9 (24), 4168-4174.

(31) Onses, M. S. Fabrication of nanopatterned poly(ethylene glycol) brushes by molecular transfer printing from poly(styreneblock-methyl methacrylate) films to generate arrays of $\mathrm{Au}$ nanoparticles. Langmuir 2015, 31 (3), 1225-1230.

(32) Goodberlet, J. G.; Hastings, J. T.; Smith, H. I. Performance of the Raith 150 electron-beam lithography system. J. Vac. Sci. Technol., B: Microelectron. Process. Phenom. 2001, 19 (6), 2499-2503.

(33) Onses, M. S.; Liu, C. C.; Thode, C. J.; Nealey, P. F. Highly selective immobilization of $\mathrm{Au}$ nanoparticles onto isolated and dense nanopatterns of poly(2-vinyl pyridine) brushes down to single-particle resolution. Langmuir 2012, 28 (18), 7299-7307.

(34) Mansky, P.; Liu, Y.; Huang, E.; Russell, T. P.; Hawker, C. J. Controlling polymer-surface interactions with random copolymer brushes. Science 1997, 275 (5305), 1458-1460.

(35) Torun, I.; Celik, N.; Hancer, M.; Es, F.; Emir, C.; Turan, R.; Onses, M. S. Water Impact Resistant and Antireflective Superhydrophobic Surfaces Fabricated by Spray Coating of Nanoparticles: Interface Engineering via End-Grafted Polymers. Macromolecules 2018, 51 (23), 10011-10020.

(36) Kim, M.; Schmitt, S. K.; Choi, J. W.; Krutty, J. D.; Gopalan, P. From Self-assembled monolayers to coatings: advances in the synthesis and nanobio applications of polymer brushes. Polymers 2015, 7 (7), 1346-1378.

(37) Zdyrko, B.; Varshney, S. K.; Luzinov, I. Effect of molecular weight on synthesis and surface morphology of high-density poly(ethylene glycol) grafted layers. Langmuir 2004, 20 (16), 6727-6735.

(38) Liu, K. J.; Parsons, J. L. Solvent effects on the preferred conformation of poly (ethylene glycols). Macromolecules 1969, 2 (5), $529-533$.

(39) Biswas, S.; Liu, X.; Jarrett, J. W.; Brown, D.; Pustovit, V.; Urbas, A.; Knappenberger, K. L., Jr; Nealey, P. F.; Vaia, R. A. Nonlinear chiro-optical amplification by plasmonic nanolens arrays formed via directed assembly of gold nanoparticles. Nano Lett. 2015, 15 (3), 1836-1842.

(40) Zheng, Y.; Rosa, L.; Thai, T.; Ng, S. H.; Gómez, D. E.; Ohshima, H.; Bach, U. Asymmetric gold nanodimer arrays: electrostatic self-assembly and SERS activity. J. Mater. Chem. A 2015, 3 (1), 240-249. 
(41) Akhtar, M. S.; Panwar, J.; Yun, Y. S. Biogenic synthesis of metallic nanoparticles by plant extracts. ACS Sustainable Chem. Eng. 2013, 1 (6), 591-602.

(42) Bahram, M.; Mohammadzadeh, E. Green synthesis of gold nanoparticles with willow tree bark extract: a sensitive colourimetric sensor for cysteine detection. Anal. Methods 2014, 6 (17), 69166924.

(43) Sarwar, R.; Farooq, U.; Raza Shah, M.; Khan, S.; Riaz, N.; Naz, S.; Ibrar, A.; Khan, A. Rapid synthesis of gold nanoparticles from quercus incana and their antimicrobial potential against human pathogens. Appl. Sci. 2017, 7 (1), 29.

(44) Bastus, N. G.; Merkoçi, F.; Piella, J.; Puntes, V. Synthesis of highly monodisperse citrate-stabilized silver nanoparticles of up to $200 \mathrm{~nm}$ : kinetic control and catalytic properties. Chem. Mater. 2014, 26 (9), 2836-2846.

(45) Karabel Ocal, S.; Patarroyo, J.; Kiremitler, N. B.; Pekdemir, S.; Puntes, V. F.; Onses, M. S. Plasmonic Assemblies of Gold Nanorods on Nanoscale Patterns of Poly(ethylene glycol): Application in Surface-Enhanced Raman Spectroscopy. J. Colloid Interface Sci. 2018, 532, 449-455.

(46) Nugroho, A.; Song, B. M.; Seong, S. H.; Choi, J. S.; Choi, J.; Choi, J. Y.; Park, H. J. HPLC analysis of phenolic substances and antialzheimer's activity of Korean quercus species. Nat. Prod. Sci. 2016, 22 (4), 299-306.

(47) Judy, J. D.; Tollamadugu, N. P.; Bertsch, P. M. Pin oak (Quercus palustris) leaf extract mediated synthesis of triangular, polyhedral and spherical gold nanoparticles. Adv. Nanopart. 2012, 01 (03), 79-85.

(48) Park, J. W.; Shumaker-Parry, J. S. Structural study of citrate layers on gold nanoparticles: role of intermolecular interactions in stabilizing nanoparticles. J. Am. Chem. Soc. 2014, 136 (5), 19071921.

(49) Sun, H.; Liu, H.; Wu, Y. A green, reusable SERS film with high sensitivity for in-situ detection of thiram in apple juice. Appl. Surf. Sci. 2017, 416, 704-709.

(50) Le Ru, E. C.; Blackie, E.; Meyer, M.; Etchegoin, P. G. Surface enhanced Raman scattering enhancement factors: A comprehensive study. J. Phys. Chem. C 2007, 111 (37), 13794-13803.

(51) Yilmaz, M.; Babur, E.; Ozdemir, M.; Gieseking, R. L.; Dede, Y.; Tamer, U.; Schatz, G. C.; Facchetti, A.; Usta, H.; Demirel, G. Nanostructured organic semiconductor films for molecular detection with surface-enhanced Raman spectroscopy. Nat. Mater. 2017, 16, 918-924.

(52) Wang, Y.; Wang, M.; Shen, L.; Sun, X.; Shi, G.; Ma, W.; Yan, X. High-performance flexible surface-enhanced Raman scattering substrates fabricated by depositing $\mathrm{Ag}$ nanoislands on the dragonfly wing. Appl. Surf. Sci. 2018, 436, 391-397.

(53) Kim, J. Y.; Kim, H.; Kim, B. H.; Chang, T.; Lim, J.; Jin, H. M.; Mun, J. H.; Choi, Y. J.; Chung, K.; Shin, J. Highly tunable refractive index visible-light metasurface from block copolymer self-assembly. Nat. Commun. 2016, 7, 12911.

(54) Sakir, M.; Pekdemir, S.; Karatay, A.; Küçüköz, B. 1.; Ipekci, H. H.; Elmali, A.; Demirel, G.; Onses, M. S. Fabrication of plasmonically active substrates using engineered silver nanostructures for SERS applications. ACS Appl. Mater. Interfaces 2017, 9 (45), 39795-39803. (55) Li, Y.; Dykes, J.; Gilliam, T.; Chopra, N. A new heterostructured SERS substrate: free-standing silicon nanowires decorated with graphene-encapsulated gold nanoparticles. Nanoscale 2017, 9 (16), 5263-5272.

(56) Lin, D.; Wu, Z.; Li, S.; Zhao, W.; Ma, C.; Wang, J.; Jiang, Z.; Zhong, Z.; Zheng, Y.; Yang, X. Large-area Au-nanoparticle-functionalized $\mathrm{Si}$ nanorod arrays for spatially uniform surface-enhanced Raman spectroscopy. ACS Nano 2017, 11 (2), 1478-1487.

(57) Zou, X.; Wang, Y.; Liu, W.; Chen, L. m-Cresol Purple Functionalized Surface Enhanced Raman Scattering Paper Chips for Highly Sensitive Detection of $\mathrm{pH}$ in the Neutral $\mathrm{pH}$ Range. Analyst 2017, 142, 2333-2337.

(58) Cui, Y.; Phang, I. Y.; Lee, Y. H.; Lee, M. R.; Zhang, Q.; Ling, X. Y. Multiplex plasmonic anti-counterfeiting security labels based on surface-enhanced Raman scattering. Chem. Commun. 2015, 51 (25), $5363-5366$

(59) Lee, M. R.; Lee, H. K.; Yang, Y.; Koh, C. S. L.; Lay, C. L.; Lee, Y. H.; Phang, I. Y.; Ling, X. Y. Direct Metal Writing and Precise Positioning Gold of Nanoparticles within Microfluidic Channels for SERS Sensing of Gaseous Analytes. ACS Appl. Mater. Interfaces 2017, 9 (45), 39584-39593.

(60) Liu, H.; Qian, X.; Wu, Z.; Yang, R.; Sun, S.; Ma, H. Microfluidic Synthesis of QD-Encoded PEGDA Microspheres for Suspension Assay. J. Mater. Chem. B 2016, 4, 482-488.

(61) Cashman, J. N. The mechanisms of action of NSAIDs in analgesia. Drugs 1996, 52, 13-23.

(62) Kosjek, T.; Heath, E.; Krbavčič, A. Determination of nonsteroidal anti-inflammatory drug (NSAIDs) residues in water samples. Environ. Int. 2005, 31 (5), 679-685.

(63) Gros, M.; Petrović, M.; Ginebreda, A.; Barceló, D. Removal of pharmaceuticals during wastewater treatment and environmental risk assessment using hazard indexes. Environ. Int. 2010, 36 (1), 15-26.

(64) Ahmed, M. B.; Zhou, J. L.; Ngo, H. H.; Guo, W.; Thomaidis, N. S.; $\mathrm{Xu}, \mathrm{J}$. Progress in the biological and chemical treatment technologies for emerging contaminant removal from wastewater: a critical review. J. Hazard. Mater. 2017, 323, 274-298.

(65) Nir, R. R.; Nahman-Averbuch, H.; Moont, R.; Sprecher, E.; Yarnitsky, D. Preoperative preemptive drug administration for acute postoperative pain: A systematic review and meta-analysis. Eur. J. Pain 2016, 20 (7), 1025-1043. 